\title{
Aarco Dificuldades operacionais no uso de coleiras caninas impregnadas com inseticida para o controle da leishmaniose visceral, Montes Claros, MG, 2012*
}

\author{
Operational difficulties in the use of insecticidal dog collars for the control of visceral \\ leishmaniasis, municipality of Montes Claros, MG, Brazil, 2012
}

\section{Dificultades operativas en el uso de collares impregnados con insecticida para el control de la leishmaniasis visceral, municipio de Montes Claros, MG, Brasil, 2012}

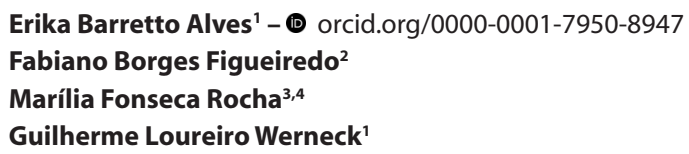

'Universidade Federal do Rio de Janeiro, Instituto de Estudos em Saúde Coletiva, Programa de Pós-Graduação em Saúde Coletiva, Rio de Janeiro, RJ, Brasil

${ }^{2}$ Fundação Instituto Oswaldo Cruz, Instituto Carlos Chagas, Laboratório de Biologia Celular, Curitiba, PR, Brasil ${ }^{3}$ Universidade Estadual de Montes Claros, Centro de Ciências Biológicas e da Saúde, Departamento de Saúde Mental e Saúde Coletiva, Montes Claros, MG, Brasil

${ }^{4}$ Secretaria Municipal de Saúde, Centro de Controle de Zoonoses, Programa de Controle das Leishmanioses, Montes Claros, MG, Brasil

\section{Resumo}

Objetivo: descrever dificuldades operacionais na implementação de coleiras caninas impregnadas com deltametrina para o controle da leishmaniose visceral. Métodos: estudo de intervenção comunitária no município de Montes Claros, MG, Brasil, comparando (i) área-controle - sem encoleiramento canino - e (ii) área de intervenção - uso de coleiras caninas impregnadas com deltametrina 4\%; foi realizado inquérito sorológico inicial, seguido de três outros ciclos (aos 12, 18 e 24 meses). Resultados: de 4.388 cães inicialmente soronegativos encoleirados, 36,9\% não foram encontrados no segundo ciclo, $27,0 \%$ foram perdidos por sumiço/doação/venda e $22,6 \%$ porque a casa estava fechada; a perda de coleiras foi de $56,1 \%$ em um ano; entre os cães que permaneceram mais tempo no estudo, a perda foi menor. Conclusão: as altas frequências de perda de coleiras e de domicílios fechados são dificuldades operacionais para a implementação de um programa de controle baseado na estratégia avaliada.

Palavras-chave: Leishmaniose Visceral; Leishmania infantum; Cães; Prevenção de Doenças; Seguimentos.

\footnotetext{
* Artigo derivado de tese de Doutorado intitulada 'Avaliação da efetividade do uso das coleiras impregnadas com inseticida para o controle da leishmaniose visceral no município de Montes Claros, MG', defendida por Erika Barretto Alves junto ao Programa de Pós-Graduação em Saúde Coletiva da Universidade Federal do Rio de Janeiro (UFRJ) em 2016. O projeto de pesquisa foi financiado pelo Ministério da Saúde (Convênio n 01234/2010) e pela Organização Pan-Americana da Saúde (Carta Acordo BR/LOA/1300025.001). Para sua realização, o autor Guilherme Loureiro Werneck recebeu bolsa de produtividade do Conselho Nacional de Desenvolvimento Científico e Tecnológico (CNPq:Processo no 311507/2014-0).
}

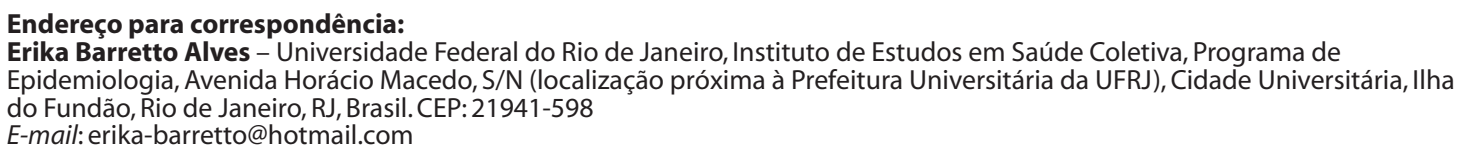




\section{Introdução}

Nas Américas, a leishmaniose visceral (LV) é uma doença causada por protozoários da espécie Leishmania infantum, transmitida pela picada de flebotomíneos, sendo Lutzomyia longipalpis a espécie de maior importância epidemiológica. Destaca-se sua facilidade de adaptação ao ambiente urbano e periurbano, assim como o aumento de sua densidade populacional após períodos chuvosos. ${ }^{1-3}$

0 cão doméstico tem sido indicado como principal reservatório em meio urbano. ${ }^{3}$ Há evidências da correlação espacial entre a incidência de casos humanos e a prevalência da infecção em cães de áreas urbanas, geralmente com a infecção canina a preceder os casos humanos. ${ }^{4,5}$

A LV é uma doença tropical negligenciada, não obstante sua relevância no cenário global. ${ }^{3,6} 0$ rótulo de doença tipicamente rural já foi superado e seu ciclo está estabelecido em áreas urbanas e periurbanas. ${ }^{7}$ Estima-se que anualmente, ocorram entre 0,2 e 0,4 milhão de casos de LV no mundo ${ }^{6}$ e que, no ano de 2015, 90\% desses casos tenham se concentrado em apenas sete países: Índia, Quênia, Somália, Sudão, Sudão do Sul, Etiópia e Brasil. ${ }^{8}$

\section{0 cão doméstico tem sido indicado como principal reservatório em meio urbano. Há evidências da correlação espacial entre a incidência de casos humanos ea prevalência da infecção em cães de áreas urbanas, geralmente com a infeç̧ão canina a preceder os casos humanos.}

0 processo de urbanização da doença no Brasil teve início na década de 1980, notadamente nas cidades de Teresina, PI, São Luís, MA, e Montes Claros, MG, culminando com a instalação definitiva da LV em cidades de médio e grande porte. ${ }^{2,5,9}$

0 controle da doença no país, orientado pelo Ministério da Saúde, está pautado em três estratégias: diagnóstico precoce e tratamento dos casos humanos; redução da população de flebotomíneos; e eliminação dos reservatórios domésticos. ${ }^{5}$ Todavia, essas medidas têm se mostrado pouco efetivas para conter a disseminação da doença no território nacional. ${ }^{10} \mathrm{~A}$ remoção dos cães infectados persiste como uma medida controversa, sendo insuficientes as evidências científicas disponíveis e sua efetividade para reduzir a incidência da doença. ${ }^{10,11}$ Werneck et al. ${ }^{11}$ ressaltam que, após mais de 40 anos de existência do Programa de Vigilância e Controle da Leishmaniose Visceral (PVCLV), a eliminação de cães em larga escala não está sendo bem-sucedida no controle da LV, demandando uma reavaliação profunda das estratégias vigentes. Ademais, as intervenções preconizadas enfrentam sérias dificuldades, de caráter logístico e financeiro, para sua implementação., ${ }^{2,12}$ Há, contudo, autores que defendem um rastreamento sorológico canino sistemático, acompanhado de eutanásia dos animais positivos, por considerarem ser uma boa estratégia de controle, capaz de uma redução contundente na prevalência de infecção canina e dos casos humanos. ${ }^{13,14}$

Por sua vez, estudos avaliativos de medidas de controle alternativas, como o uso de coleiras caninas impregnadas com deltametrina 4\%, têm demonstrado resultados satisfatórios. ${ }^{15,16}$ Esse dispositivo, além de evitar a picada dos flebotomíneos, aumenta a mortalidade desses insetos, reduzindo a circulação da $L$. infantum em locais onde o cão é o principal reservatório do parasito. ${ }^{17}$ Trata-se, também, de estratégia de implementação mais simples, de mais fácil aceitação pela população, quando comparada à eliminação de cães infectados. ${ }^{17}$ Entretanto, são poucos os estudos no país sobre a efetividade desse tipo de intervenção. Em Andradina, SP, um estudo mostrou a efetividade das coleiras na queda da prevalência canina e da incidência de casos humanos, quando associadas às medidas de controle vigentes. ${ }^{18}$ Outro estudo, ao comparar a efetividade das atuais estratégias de controle, por meio de simulações, observou o melhor desempenho no uso das coleiras impregnadas que na eutanásia e na vacinação. ${ }^{6,19}$

Embora já tenha apresentado resultados satisfatórios, a implementação em massa das coleiras impregnadas com deltametrina vem acompanhada de algumas dificuldades operacionais, as quais, se não contornadas, podem comprometer o impacto da intervenção. Estudos apontaram que perdas de coleira e baixa cobertura do encoleiramento são problemas a serem enfrentados, evitando-se que reduzam a efetividade da ação. ${ }^{6,19,15}$ É fundamental conhecer os obstáculos à utilização desses dispositivos como medida de controle em Saúde Pública, para a redução da prevalência da leishmaniose visceral canina (LVC). 0 presente artigo tem como objetivo descrever as dificuldades operacionais na implementação de coleiras caninas impregnadas com deltametrina $4 \%$ para o controle da leishmaniose visceral. 


\section{Métodos}

Trata-se de um estudo de intervenção comunitária realizado no município de Montes Claros, estado de Minas Gerais, Brasil, no período de agosto de 2012 a janeiro de 2015.

Montes Claros conta com uma população de 361.915 habitantes majoritariamente urbana (95\%), segundo o Censo 2010, e está situada ao norte do estado, a $422 \mathrm{~km}$ da capital Belo Horizonte..$^{20}$ A região apresenta clima quente e seco, e predomínio de vegetação típica do cerrado, além da caatinga. A economia local é baseada principalmente na pecuária de corte e leite, seguida pela agricultura, ${ }^{21}$ e 0 valor médio do rendimento mensal domiciliar per capita era de $\mathrm{R} \$ 568,00 \mathrm{em} 2010 .^{20}$

Montes Claros se configura como uma área endêmica para LV e entre seus residentes, de 2007 a 2013, foram registrados 168 casos de $\mathrm{LV}$, dos quais $23 \mathrm{em}$ 2013 , colocando a cidade na $19^{\mathrm{a}}$ posição entre as cidades brasileiras com maior número de casos humanos da doença. ${ }^{22}$

Conforme mostra a Figura 1, o estudo se baseou na comparação entre duas áreas do município com características socioeconômicas e ambientais similares, aleatoriamente alocadas para duas estratégias de controle: (i) área-controle, sem implementação das coleiras caninas impregnadas com deltametrina $4 \%$ como medida de controle; e (ii) área de intervenção, onde foram aplicadas coleiras caninas impregnadas com deltametrina $4 \%$ como medida de controle. Ambas as áreas somavam, aproximadamente, $30 \mathrm{mil}$ habitantes e 6 mil cães. A área de intervenção englobava 15 bairros com registro de dez casos humanos de IV entre 2009 e 2011. Com o mesmo número de bairros, a área-controle registrou 12 casos humanos de LV para o mesmo triênio.

No início do estudo, em setembro e outubro de 2012, foi realizado nas duas áreas (controle e intervenção) um inquérito sorológico censitário entre cães domiciliados para detecção da prevalência de LVC. Foram planejados outros inquéritos censitários para o monitoramento da infecção canina em três ciclos subsequentes ao primeiro inquérito - aos 6 , 12 e 18 meses -, acompanhados de eutanásia dos cães infectados. Contudo, o segundo ciclo só foi implementado um ano após a etapa inicial, devido a problemas operacionais na distribuição das coleiras. 0 monitoramento da infecção canina (áreas-controle e de intervenção) e a substituição das coleiras (área de intervenção) aconteceram com base em inquéritos censitários aos 12, 18 e 24 meses após o inquérito inicial. Todos os cães das áreas sob estudo eram elegiveis para participar dos inquéritos, desde que obtida a autorização do proprietário.

$\mathrm{Na}$ área de intervenção, coleiras impregnadas com deltametrina $4 \%$ foram disponibilizadas para serem colocadas em todos os cães participantes do inquérito inicial e dos subsequentes, independentemente da condição sorológica dos animais. Na área de intervenção, a cada ciclo as coleiras eram substituídas; não houve substituição nos períodos entre os ciclos. A empresa fabricante das coleiras encarregou-se do treinamento das equipes do estudo para o correto encoleiramento.

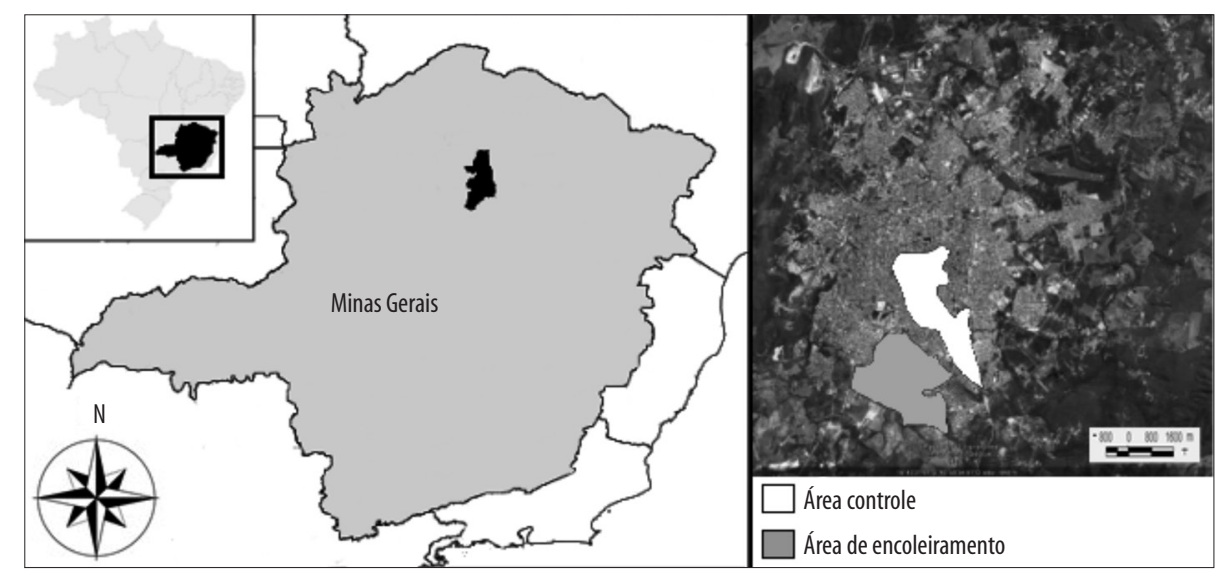

Figura 1 - Áreas selecionadas para 0 estudo de intervenção comunitária com implementação de coleiras caninas impregnadas com deltametrina 4\%, Montes Claros, MG, 2012-2015 
Profissionais treinados e sob supervisão de médico veterinário iam às casas e, ao identificarem um cão, pediam autorização ao proprietário para participação do animal no projeto. Caso consentisse, o proprietário assinava um Termo de Consentimento Livre e Esclarecido.

Na primeira participação do cão no estudo, uma ficha era preenchida com os seguintes dados: sexo (macho; fêmea), idade (em anos), raça (pura; não pura) e tempo de residência do cão no domicílio (em anos: até um; entre um e dois; mais de dois). Na área sob intervenção, procedeu-se ao encoleiramento dos animais. Nos inquéritos subsequentes, eram preenchidas fichas com as seguintes informações: se 0 proprietário aceitou participar da pesquisa; e se 0 animal estava encoleirado no momento da visita (área de intervenção). No momento da visita, se a casa fosse encontrada fechada, uma segunda tentativa era feita, em dia e horário diferentes. Novos cães podiam ser incorporados ao estudo em qualquer dos quatro ciclos, como também podiam deixar de participar; e até retornar, em ciclo posterior. Os cães soropositivos não recolhidos para eutanásia podiam permanecer no estudo e, para aqueles da área de intervenção, receber a coleira. Não houve substituição de coleiras entre os ciclos.

No rastreamento de infecção por $L$. infantum, retirou-se amostra de sangue por punção na região auricular distal para realização, em campo, do ensaio imunocromatográfico Dual-Path Platform (DPP®) (Bio-Manguinhos/Fiocruz, Rio de Janeiro, RJ, Brasil), com resultado obtido em cerca de 15 minutos. Animais com DPP reativo foram submetidos a venopunção da veia cefálica ou jugular para coleta de cerca de $2 \mathrm{~mL}$ de sangue, armazenados em tubo sem anticoagulante. As amostras foram transportadas para o laboratório Macrorregional da Superintendência Regional de Saúde de Montes Claros, sob refrigeração, e o soro separado por centrifugação (rotação de 3.000rpm, por 10 minutos) e conservado a $-20^{\circ} \mathrm{C}$ até 0 momento da realização do teste sorológico confirmatório. Na confirmação de positividade para infecção por $L$. infantum nas amostras de soro, foi empregado o teste ELISA (enzyme-linked immunosorbent assay), realizado de acordo com as recomendações do fabricante. 0 cão foi considerado infectado quando apresentou um resultado positivo de DPP, seguido de ELISA reativo, conforme recomendação do Ministério da Saúde. 5

Os animais infectados foram recolhidos e encaminhados para eutanásia, realizada sob supervisão de veterinário do Centro de Controle de Zoonoses (CCZ) do município, segundo as normas éticas preconizadas pelo Conselho Federal de Medicina Veterinária. Os procedimentos para a realização de exames sorológicos caninos e para o recolhimento dos cães soropositivos foram realizados segundo a rotina do CCZ, inclusive com o uso de equipamentos de proteção individual e focinheiras para os cães. Nos casos em que o proprietário do animal infectado se recusou a entregá-lo para eutanásia, o cão não foi excluído do estudo, podendo ser encoleirado (se pertencesse à área de intervenção) e participar dos inquéritos subsequentes.

Cada proprietário foi instruído a identificar eventuais reações alérgicas após a colocação da coleira. Na ocorrência dessas reações, o proprietário deveria entrar em contato imediato, por chamada telefônica gratuita, com a central de atendimento ao consumidor da empresa responsável pelo produto, para obter orientações de médico veterinário. As visitas ocorreram entre agosto de 2012 e janeiro de 2015.

Os dados foram consolidados em tabelas descritivas das características das áreas de estudo, participação dos cães nos diferentes ciclos, frequência e motivos para perdas de seguimento, ademais da frequência de perda de coleiras entre ciclos. Avaliaram-se as diferenças na distribuição das características dos cães segundo a área (controle ou intervenção), pelo teste de qui-quadrado de Pearson (proporções) ou teste $\mathrm{t}$ de Student (médias), com nível de significância de 5\%.

0 projeto foi aprovado pela Comissão de Ética no Uso de Animais da Fundação Instituto Oswaldo Cruz (Licença LW-70/12) e considerado isento de necessidade de avaliação ética para estudos em humanos pelo Comitê de Revisão Ética da Organização Pan-Americana da Saúde (Referência: PAHO-2012-11-0024). Conforme previsto no protocolo do estudo, no último ciclo de visitas, todos os cães-inclusive os da área-controle-foram encoleirados.

\section{Resultados}

Participaram de pelo menos um dos ciclos do estudo 20.477 cães, dos quais $54,2 \%$ da área de intervenção. Mais da metade dos cães participou de apenas um ciclo, $17,5 \%$ dos cães estiveram em dois ciclos consecutivos, e 12,7\% em três ciclos consecutivos (Tabela 1).

Dos 9.002 cães que participaram do inquérito inicial, 54,3\% eram da área de intervenção. No grupo encoleirado, era maior a presença de animais de pêlo curto $(\mathrm{p}=0,019)$, sem raça definida $(\mathrm{p}<0,001)$ e com 
Tabela 1 - Distribuição dos cães segundo participação nos ciclos e área de encoleiramento, Montes Claros, MG, 2012-2015

\begin{tabular}{|c|c|c|c|c|c|c|c|c|c|c|}
\hline \multirow{2}{*}{ Número de ciclos } & \multicolumn{4}{|c|}{ Participação nos ciclos } & \multicolumn{2}{|c|}{ Controle } & \multicolumn{2}{|c|}{ Intervenção } & \multicolumn{2}{|c|}{ Total } \\
\hline & 1 & 2 & 3 & 4 & $\mathbf{n}$ & $\%$ & $\mathbf{n}$ & $\%$ & N & $\%$ \\
\hline \multirow{4}{*}{1 ciclo } & $x$ & . & . & . & 1.675 & 17,9 & 1.815 & 16,3 & \multirow{4}{*}{10.555} & \multirow{4}{*}{51,5} \\
\hline & . & $x$ & . & . & 981 & 10,5 & 1.063 & 9,6 & & \\
\hline & . & . & $x$ & . & 840 & 9,0 & 1.055 & 9,5 & & \\
\hline & . & . & . & $\mathrm{X}$ & 1.467 & 15,6 & 1.659 & 14,9 & & \\
\hline \multirow{6}{*}{2 ciclos } & $x$ & $x$ & . & . & 483 & 5,1 & 603 & 5,4 & \multirow{6}{*}{4.213} & \multirow{6}{*}{20,6} \\
\hline & . & $x$ & $x$ & . & 528 & 5,6 & 506 & 4,6 & & \\
\hline & . & . & $x$ & $x$ & 584 & 6,2 & 876 & 7,9 & & \\
\hline & $x$ & . & $x$ & . & 107 & 1,1 & 94 & 0,9 & & \\
\hline & $x$ & . & . & $x$ & 61 & 0,6 & 59 & 0,5 & & \\
\hline & . & $x$ & . & $\mathrm{X}$ & 140 & 1,5 & 172 & 1,5 & & \\
\hline \multirow{4}{*}{3 ciclos } & $x$ & $x$ & $x$ & . & 477 & 5,1 & 516 & 4,6 & \multirow{4}{*}{3.205} & \multirow{4}{*}{15,7} \\
\hline & . & $x$ & $X$ & $x$ & 717 & 7,7 & 887 & 8,0 & & \\
\hline & $X$ & $x$ & . & $X$ & 166 & 1,8 & 200 & 1,8 & & \\
\hline & $x$ & . & $x$ & $x$ & 136 & 1,5 & 106 & 1,0 & & \\
\hline \multirow[t]{2}{*}{4 ciclos } & $X$ & $x$ & $x$ & $X$ & 1.009 & 10,8 & 1.495 & 13,5 & 2.504 & 12,2 \\
\hline & & & & Total & 9.371 & 100,0 & 11.106 & 100,0 & 20.477 & 100,0 \\
\hline
\end{tabular}

Legenda:

$X=$ participou do ciclo.

= não participou do ciclo.

menor tempo de moradia na residência $(\mathrm{p}<0,001)$. $\mathrm{Na}$ área-controle, a média de cães por domicílio foi de 1,62 - próxima à da área sob intervenção, de 1,59 cão por domicílio $(\mathrm{p}=0,403)$. As áreas não apresentaram diferença em relação às médias de idade dos cães e proporção de fêmeas. 0 intervalo de tempo médio entre a primeira e a segunda visitas foi discretamente maior na área sem intervenção, 357 dias (desvio-padrão=11), quando comparado ao correspondente intervalo de tempo na área com encoleiramento, 349 dias (desvio-padrão=14), $(\mathrm{p}<0,001)$. Não houve diferença significativa entre as duas áreas quanto à prevalência de infecção no inquérito inicial $(\mathrm{p}=0,732)$, com quase $10 \%$ de animais soropositivos (Tabela 2 ).

Dos 875 animais positivos resultantes do inquérito inicial, 670 (76,6\%) foram eutanasiados pelo CCZ: 301 na área-controle (74,7\%) e 369 na área de intervenção $(78,2 \%)(\mathrm{p}=0,225)$ (Tabela 3).

Conforme apresentado na Tabela 3, dos 403 cães soropositivos identificados na área-controle, 102 $(25,3 \%)$ não foram eutanasiados pelo CCZ por razões diversas: principalmente pela recusa do proprietário a entregar seu cão para eutanásia, ou por morte do animal antes do recolhimento. Já na área com intervenção, dos 472 cães soropositivos, 103 (21,8\%) não foram recolhidos, e assim como na área-controle, o motivo principal foi a recusa do proprietário a entregar seu cão para eutanásia, seguido da morte do animal.

Dos cães soronegativos que compuseram a linha de base do estudo, 39,6\% não estiveram no segundo ciclo; destes, 29,8\% não participaram sob a alegação de óbito pelo proprietário, e 27,2\% pelo fato de a casa estar fechada no momento da visita. Na área onde houve intervenção, o óbito do cão - por qualquer causa - foi a razão mais frequente para sua saída do estudo no segundo ciclo (30,1\%); a alegação de que o cão sumiu, foi doado ou vendido aparece em seguida, com $27,0 \%$. Já na área-controle, a casa fechada foi a principal justificativa para a descontinuidade na participação do cão no projeto, com 31,8\%, seguida da morte do animal, com 29,5\% (Tabela 4).

No primeiro ciclo, 99,9\% dos cães da área de intervenção foram encoleirados. Mesmo com um intervalo de aproximadamente um ano, a perda de coleiras entre o primeiro e o segundo ciclos do estudo foi de $56,1 \%$, valor não muito superior às perdas em ciclos 
Tabela 2 - Características dos cães, prevalência de infecção e proporção de cães soropositivos recolhidos por área de encoleiramento, Montes Claros, MG, 2012-2015

\begin{tabular}{|c|c|c|c|}
\hline Variável & Controle & Intervenção & Valor de $\mathrm{p}^{\mathrm{a}}$ \\
\hline \multicolumn{4}{|l|}{ Sexo (\%) } \\
\hline Fêmea & $2.277(55,3)$ & $2.772(56,7)$ & \multirow{2}{*}{0,194} \\
\hline Macho & $1.837(44,7)$ & $2.116(43,3)$ & \\
\hline \multicolumn{4}{|l|}{ Raça pura (\%) } \\
\hline Não & $2.259(54,9)$ & $3.056(62,5)$ & \multirow{2}{*}{$<0,001$} \\
\hline Sim & $1.855(45,1)$ & $1.832(37,5)$ & \\
\hline \multicolumn{4}{|l|}{ Pêlo (\%) } \\
\hline Longo & $1.246(30,3)$ & $1.353(27,7)$ & \multirow{2}{*}{0,019} \\
\hline Curto & $2.866(69,7)$ & $3.534(72,3)$ & \\
\hline \multicolumn{4}{|l|}{ Tempo na residência em anos (\%) } \\
\hline$<1$ & $855(20,8)$ & $1.450(29,7)$ & \multirow{3}{*}{$<0,001$} \\
\hline $1-2$ & $1.163(28,3)$ & $1.007(20,6)$ & \\
\hline$>2$ & $2.095(50,9)$ & $2.427(49,7)$ & \\
\hline \multicolumn{4}{|l|}{ Cão positivo recolhido (\%) } \\
\hline Não & $102(25,3)$ & $103(21,8)$ & \multirow{2}{*}{0,225} \\
\hline Sim & $301(74,7)$ & $369(78,2)$ & \\
\hline Infecção (\%) & $403(9,9)$ & $472(9,7)$ & 0,732 \\
\hline Média de idade em anos (DPb) & $3,4(3,2)$ & $3,4(3,0)$ & 0,673 \\
\hline Média de cães por domicílio (DPb) & $1,6(1,1)$ & $1,6(1,0)$ & 0,403 \\
\hline
\end{tabular}

a) Teste qui-quadrado de Pearson (variáveis categóricas) ou teste $t$ de Student (diferença de médias de variáveis contínuas).

b) DP: desvio-padrão.

Tabela 3 - Destino dos cães sororreagentes no primeiro ciclo segundo área de encoleiramento, Montes Claros, MG, Brasil, 2012-2015

\begin{tabular}{lccccc}
\hline \multirow{2}{*}{ Cães sororreagentes } & \multicolumn{2}{c}{ Controle } & \multicolumn{3}{c}{ Intervenção } \\
\cline { 2 - 5 } \cline { 3 - 5 } & $\mathbf{n}$ & $\%$ & $\mathbf{n}$ & 369 & 78,2 \\
\hline Recolhidos & 301 & 16,1 & 69 & 14,6 \\
Recusa & 65 & 2,5 & 23 & 4,9 \\
Morte & 10 & 0,7 & 4 & 0,8 \\
Sumiu/doado/vendido & 3 & 0,3 & - & 0,0 \\
Proprietário se mudou & 1 & 5,7 & 7 & 1,5 \\
Não informado & 23 & 100,0 & 472 & 100,0 \\
\hline Total & 403 & & & \\
\hline
\end{tabular}

consecutivos posteriores, de 51,2\%. A perda de coleira entre as duas primeiras avaliações (consecutivas) do cão foi de 54,0\%; entre a segunda e a terceira, de 40,8\%; e entre a terceira e a quarta avaliações, de $35,3 \%$. A perda do dispositivo entre os dois primeiros ciclos foi maior em cães com idade menor ou igual a 1 ano (65,7\%), quando comparados àqueles com idade superior a um ano $(53,5 \%)(\mathrm{p}<0,001)$.

\section{Discussão}

Tendo por base um estudo de intervenção comunitária, este artigo descreve as dificuldades operacionais na implementação de coleiras caninas impregnadas com deltametrina $4 \%$ para o controle da leishmaniose visceral. Entre os principais problemas identificados, salientam-se as altas frequências de perda de coleiras 
Tabela 4 - Perda de seguimento dos cães soronegativos entre o primeiro e o segundo ciclos, Montes Claros, MG, 2012-2015

\begin{tabular}{lccccc}
\hline \multirow{2}{*}{ Perda de cães soronegativos } & \multicolumn{2}{c}{ Controle } & \multicolumn{3}{c}{ Intervenção } \\
\cline { 2 - 5 } \cline { 3 - 5 } Casa fechada & $\mathbf{n}$ & 31,8 & $\mathbf{n}$ & \% \\
Morte & 498 & 29,5 & 367 & 30,1 \\
Sumiu/doado/vendido & 462 & 20,1 & 488 & 27,0 \\
Proprietário se mudou & 314 & 10,6 & 437 & 13,9 \\
Recusa & 166 & 3,3 & 226 & 1,9 \\
Recolhido por outro motivo & 52 & 0,6 & 30 & 0,6 \\
Difícil contenção & 10 & 0,4 & 10 & 0,6 \\
Outros & 6 & 0,4 & 10 & 0,9 \\
Não informada & 6 & 3,3 & 14 & 2,4 \\
\hline Total & 51 & 100,0 & 39 & 100,0 \\
\hline
\end{tabular}

e de domicílios fechados por ocasião da visita dos profissionais. Estas dificuldades são aspectos centrais a serem considerados, na eventualidade da implementação desse tipo de intervenção em larga escala no país.

Dos animais com infecção no primeiro ciclo, $76,6 \%$ foram eutanasiados. 0 dado é consistente com os resultados de outros estudos que também identificaram o recolhimento para eutanásia como a principal razão para a perda de seguimento de cães soropositivos. ${ }^{23-25}$ Além da eutanásia, Morais $^{23}$ apontou a alegação de morte do animal por outras causas, registrada em 5,2\% dos cães soropositivos, como outro motivo para 0 afastamento do cão do local onde residia, valor superior ao encontrado neste estudo $(2,5 \%$ na área-controle e 4,9\% na área sob intervenção).

Dos 4.053 cães que deixaram a pesquisa após o primeiro ciclo, 78,6\% eram soronegativos. A alegação de óbito do animal foi a razão mais frequente para a saída desses cães não infectados (29,8\%), valor este discretamente inferior aos 34\% identificados por Lopes no município de Juatuba, $\mathrm{MG},{ }^{24}$ no mesmo intervalo de um ano. Já Coura-Vital, ${ }^{26}$ em um seguimento de 16 meses, relatou uma perda por morte, entre cães soronegativos, de aproximadamente $6 \%$.

Encontrar a casa fechada foi o principal motivo de perda de seguimento de cães soronegativos na área-controle $(31,8 \%)$ e o terceiro mais frequente na área sob intervenção $(22,6 \%)$, uma limitação importante para a implementação do encoleiramento. Coura-Vital, ${ }^{26} \mathrm{em} 16$ meses, obteve um percentual menor de perda por esse motivo (19\%), provavelmente porque os domicílios encontrados fechados eram visitados por três vezes antes de serem considerados perdidos. É necessário repensar a estratégia de visitas, de maneira a contemplar os moradores ausentes do domicílio no horário comercial; caso contrário, eles permanecerão à margem das ações de controle preconizadas.

Deve-se destacar que a razão das perdas no estudo foi descrita a partir de relato do proprietário. Parcela dessas alegações pode representar um subterfúgio para não mais participar da pesquisa. Essa recusa não declarada poderia ser atribuída a o fato de a coleta ser realizada em animais aparentemente saudáveis, levando o proprietário a antecipar a possibilidade de um resultado positivo e potencial necessidade de eutanásia.

A perda de coleiras foi de $56 \%$, considerando-se apenas os dois primeiros encoleiramentos (consecutivos) do cão, independentemente do ciclo no qual o animal foi integrado ao estudo. Esse valor foi superior aos $41 \%$ encontrados por Reithinger et al..$^{15} \mathrm{em}$ apenas cinco meses de seguimento. Foglia-Manzillo et $a l^{27}$ relataram uma perda de $30 \%$ de coleiras em um ano, embora os cães sob estudo se encontrassem restritos ao canil. Acompanhamento de cães domiciliados no município de Andradina, SP, identificou percentuais de perdas entre avaliações semestrais bem mais baixos: $5,5 \%$ de perda no primeiro semestre de seguimento e $6,4 \%$ no último semestre. ${ }^{18}$ Reithinger et al. ${ }^{15}$ listaram algumas razões, relatadas pelos proprietários, para a perda da coleira: 0 fato de o cão conseguir soltar a fivela da coleira pouco tempo após a aplicação; o próprio desgaste do dispositivo; e eventos adversos, como irritação na pele. Vale ressaltar que foram contabilizadas perdas menores à medida que avançava o tempo do cão no estudo. Dados sugerem que 0 transcurso do tempo favorece a melhor adaptação do 
animal ao dispositivo, aspecto bastante vantajoso para um futuro programa de controle que incorpore coleiras com deltametrina. Devem-se discutir estratégias de reposição mais rápida das coleiras, cujas perdas, se em grande proporção, podem comprometer o desempenho dessa medida. No que concerne às reações, apenas 74 cães $(0,7 \%)$ foram objeto de relato de alergia à coleira e, em 14,8\% desses casos, o relato foi feito por ocasião da primeira visita, antes mesmo do encoleiramento.

0 impacto do uso das coleiras na transmissão da LVC depende das taxas de cobertura (idealmente, acima de $90 \%)^{6,19} \mathrm{e}$ da perda desses dispositivos, ${ }^{15} \mathrm{o}$ que implica a reposição oportuna das coleiras e 0 encoleiramento dos cães recém-chegados à localidade. Além do alto percentual de coleiras perdidas, uma das dificuldades capazes de comprometer a efetividade da intervenção foi a entrada de mais de 2.600 cães no segundo ciclo, na área de intervenção (quase metade dos animais que participaram deste ciclo). Isto significa que parte desses cães já poderiam habitar essa área e estariam sem coleira até 0 início desse segundo ciclo. Dos cães da área de intervenção que entraram na segunda etapa, $46 \%$ se encontravam na residência há pelo menos um ano.

Embora as duas áreas estudadas - controle e intervenção - não tenham apresentado diferenças na prevalência de infecção no inquérito inicial, sugerindo força de transmissão semelhante, foi identificada variação significativa para algumas variáveis: raça, tipo de pêlo e tempo na residência. Essas diferenças deveriam ser consideradas ao se avaliar a efetividade do uso das coleiras, pois, ao influenciarem de forma independente o risco individual de LVC, poderiam levar a erros nas estimativas de efetividade.

No planejamento inicial do presente estudo, o uso das coleiras seria avaliado conjuntamente com a eliminação dos cães positivos e a borrifação do domicílio por inseticida. Entretanto, a borrifação ocorreu em aproximadamente $1 \%$ dos domicílios, inviabilizando sua utilização nas análises. 0 uso de inseticida no combate ao flebotomíneo reduz o risco de soroconversão em cães de área endêmica e é uma medida factível de controle da doença. ${ }^{28}$ Barata et al. ${ }^{29}$ em estudo realizado no município de Governador Valadares, MG, identificaram aumento da soropositividade canina em áreas com maior densidade de $L$. longipalpis, um achado a exigir implementação mais rigorosa das medidas de controle, incluindo o uso de inseticidas de ação residual.

A principal intenção deste trabalho foi descrever um panorama referencial àincorporação das coleiras na rotina dos serviços de controle da leishmaniose visceral, com base na infraestrutura municipal existente, identificando as reais dificuldades de sua adoção, cuja eficácia na prevenção de picadas de flebotomíneos em cães já foi demonstrada em outros estudos. ${ }^{17,30}$ Camargo-Neves et al..$^{18}$ frisam que a implementação de um programa de controle para LV, utilize ou não essa nova ferramenta, depende de uma estruturação do município nesse sentido, e do rigoroso planejamento de suas ações. David et al. ${ }^{17}$ ressaltam que o trabalho de campo permite conhecer a efetividade da coleira nos cães em seu habitat natural. Contudo, para se mostrar efetiva, a medida deve proporcionar uma grande redução na incidência de infecção canina em áreas endêmicas.

Sobre o presente estudo, uma questão adicional a ser considerada refere-se à periodicidade semestral dos inquéritos realizados, diferentemente do intervalo de tempo preconizado pelo Programa de Vigilância e Controle da Leishmaniose Visceral (PVCLV), qual seja, de inquéritos anuais. ${ }^{5}$ Dado o prazo de validade das coleiras aplicadas ser de seis a oito meses, a logística na implementação das medidas de controle seria mais complexa que a adotada neste estudo: as visitas domiciliares deveriam ocorrer mais frequentemente, implicando aumento nos custos do programa. Considerando-se a importância de manter um alto grau de encoleiramento, seriafundamental vislumbrar estratégias factíveis de reposição das coleiras perdidas.

Afinal, as dificuldades operacionais e limitações identificadas neste trabalho, notadamente as altas frequências de perda de coleiras e de domicílios fechados por ocasião da visita, são possíveis de serem enfrentadas, em caso de adoção pelo PVCLV do uso dessas coleiras como medida de controle da leishmaniose visceral no Brasil.

\section{Contribuição dos autores}

Alves EB participou do processamento e análise dos dados, interpretação dos resultados e redação do manuscrito. Werneck GL participou da concepção e delineamento do estudo, análise e interpretação dos dados, discussão dos resultados e redação do manuscrito. Figueiredo FB participou da concepção e delineamento do estudo, interpretação dos resultados e revisão crítica do manuscrito. Rocha MF participou da concepção e delineamento do estudo, coleta de dados, interpretação dos resultados e revisão crítica do manuscrito. Todos os autores contribuíram na elaboração e revisão final do artigo e declararam ser responsáveis por todos os aspectos do trabalho. 


\section{Referências}

1. Lainson R, Rangel EF. Lutzomyia longipalpis and the eco-epidemiology of American visceral leishmaniasis, with particular reference to Brazil: a review. Mem Inst Oswaldo Cruz. 2005 Dec;100(8):811-27.

2. Harhay MO, Olliaro PL, Costa DL, Costa CH. Urban parasitology: visceral leishmaniasis in Brazil. Trends Parasitol. 2011 Sep;27(9):403-9.

3. World Health Organization. Control of the Leishmaniases. Report of a meeting of the WHO Expert Committee on the Control of Leishmaniases [Internet]. Geneva: World Health Organization; 2010 [cited 2017 Sep 26]. Available from: http://whqlibdoc. who.int/trs/WHO_TRS_949_eng.pdf?ua=1

4. Oliveira CL, Assunção RM, Reis IA, Proietti FA. Spatial distribution of human and canine visceral leishmaniasis in Belo Horizonte, Minas Gerais State, Brasil, 1994-1997. Cad Saúde Pública. 2001 SepOct;17(5):1231-9.

5. Ministério da Saúde. Secretaria de Vigilância em Saúde. Departamento de Vigilância Epidemiológica. Manual de vigilância e controle da leishmaniose visceral [Internet]. Brasília: Ministério da Saúde; 2006 [citado 2018 jun 21]. 120 p. Disponível em: http://bvsms.saude.gov.br/bvs/publicacoes/manual_ vigilancia_controle_leishmaniose_visceral.pdf

6. Alvar J, Vélez ID, Bern C, Herrero M, Desjeux P, Cano $\mathrm{J}$, et al. Leishmaniasis worldwide and global estimates of its incidence. PLoS One. 2012 May;7(5):e35671.

7. Dantas-Torres F, Brandão-Filho SP. Visceral leishmaniasis in Brazil: revisiting paradigms of epidemiology and control. Rev Inst Med Trop. 2006 May-Jun; 48(3):151-6.

8. World Health Organization. Global health observatory (GHO) data. Leishmaniasis, 2015. [Internet]. 2015 [cited 2017 Sep 26]. Available from: http://www.who. int/gho/neglected_diseases/leishmaniasis/en/

9. Maia-Elkhoury ANS, Alves WA, Sousa-Gomes ML, Sena JM, Luna EA. Visceral leishmaniasis in Brazil: trends and challenges. Cad Saúde Pública. 2008 Dec;24(12):2941-7.

10. Romero GA, Boelaert M. Control of visceral leishmaniasis in Latin America - a systematic review. PLoS Negl Trop Dis. 2010 Jan; 4(1):e584.

11. Werneck GL, Costa CHC, Carvalho FAA, Pires e Cruz MS, Maguire JH, Castro MC. Effectiveness of insecticide spraying and culling of dogs on the incidence of Leishmania infantum infection in humans: a cluster randomized trial in Teresina, Brazil. PLoS Negl Trop Dis. 2014 Oct 30;8(10):e3172.

12. Zuben APB, Donalísio MR. Dificuldades na execução das diretrizes do Programa de Vigilância e Controle da Leishmaniose Visceral em grandes municípios brasileiros. Cad Saúde Pública. 2016 jun;32(6):e00087415.

13. Ashford DA, David JR, Freire M, David R, Sherlock I, Eulalio MC, et al. Studies on control of visceral leishmaniasis: impact of dog control on canine and human visceral leishmaniasis in Jacobina, Bahia, Brazil. Am J Trop Med Hyg. 1998 Jul;59(1):53-7.

14. Palatnik-de-Sousa CB, Santos WR, França-Silva JC, Costa RT, Reis AB, Palatnik M, et al. Impacto of canine control on the epidemiology of canine and human visceral leishmaniasis in Brazil. Am J Trop Med Hyg. 2001 Nov;65(5):510-7.

15. Reithinger R, Coleman PG, Alexander B, Vieira EP, Assis G, Davies CR. Are insecticide-impregnated dog collars a feasible alternative to dog culling as a strategy for controlling canine visceral leishmaniasis in Brazil? Int J Parasitol. 2004 Jan;34(1):55-62.

16. Gavgani AS, Hodjati MH, Mohite H, Davies CR. Effect of insecticide-impregnated dog collars on incidence of zoonotic visceral leishmaniasis in Iranian children: a matched-cluster randomised trial. Lancet. $2002 \mathrm{Aug}$ 3;360(9330):374-9.

17. David JR, Stamm LM, Bezerra HS, Souza RN, KillickKendrick R, Lima JW. Deltamethrin-impregnated dog collars have a potent anti-feeding and insecticidal effect on Lutzomyia longipalpis and Lutzomyia migonei. Mem Inst Oswaldo Cruz. 2001 Aug;96(6):839-47.

18. Camargo-Neves VLF, Rodas LAC, Pauliquevis Junior C. Avaliação da efetividade da utilização de coleiras impregnadas com deltametrina a $4 \%$ para o controle da leishmaniose visceral americana no Estado de São Paulo: resultados preliminares. Boletim Epidemiol Paulista. 2004 dez;1(12): 7-14.

19. Sevá AP, Ovallos FG, Amaku M, Carrillo E, Moreno J, Galati EAB, et al. Canine-based strategies for prevention and control of visceral leishmaniasis in Brazil. PLos One. 2016 Jul;11(7):e0160058.

20. Instituto Brasileiro de Geografia e Estatística. Cidades, Montes Claros [Internet]. 2010 [citado 2017 set 26]. Disponível em: https://cidades.ibge.gov.br/brasil/mg/ montes-claros/panorama 
21. Prefeitura de Montes Claros (MG). Aspectos gerais [Internet]. 2017 [citado 2017 set 26]. Disponível em: http://www.montesclaros.mg.gov.br/cidade/ aspectos_gerais.htm

22. Ministério da Saúde. Secretaria de Vigilância em Saúde. Datasus. Leishmaniose visceral [Internet]. 2015. [citado 2017 set 26]. Disponível em: http://tabnet.datasus.gov.br/ cgi/deftohtm.exe?sinannet/cnv/leishvbr.def

23. Morais MHF. Avaliação das atividades de controle da leishmaniose visceral na Regional Noroeste de Belo Horizonte, 2006 a 2010 [tese]. Belo Horizonte (MG): Universidade Federal de Minas Gerais; 2011.

24. Lopes EGP. Dinâmica da transmissão da leishmaniose visceral em uma coorte de cães em Juatuba-MG, de 2010 a 2011 [tese]. Belo Horizonte (MG): Universidade Federal de Minas Gerais, 2013.

25. Gonçalves SA. Controle do reservatório canino para leishmaniose visceral, na regional noroeste de Belo Horizonte, Minas Gerais, 2006-2011 [dissertação]. Belo Horizonte (MG): Universidade Federal de Minas Gerais, 2013.

26. Coura-Vital W. Estudo epidemiológico prospectivo em cães assintomáticos infectados por Leishmania

\begin{abstract}
Objective: to describe operational difficulties in the implementation of deltamethrin-impregnated dog collars for the control of visceral leishmaniasis. Methods: this was a community intervention trial in the municipality of Montes Claros, MG, Brazil, comparing (i) control area - dogs without dog collars - and (ii) intervention area - use of $4 \%$ deltamethrin-impregnated collars; an initial serological survey was performed, followed by three further cycles (at 12, 18 and 24 months). Results: out of 4,388 dogs initially seronegative wearing collars, $36.9 \%$ were not found in the second cycle, $27.0 \%$ of them were lost owing to disappearance/given away/sale, and $22.6 \%$ because no one was at home; $56.1 \%$ of collars were lost in one year; while among dogs that stayed longer in the study, collar loss was lower. Conclusion: high frequencies of collar loss and no one being at home at the time of the visit are operational difficulties for the implementation of a national control program based on the strategy evaluated.
\end{abstract}

Keywords: Leishmaniasis, Visceral; Leishmania infantum; Dogs; Disease Prevention; Follow-up Studies.
(Leishmania) infantum e identificação de biomarcadores de infecção, 2011 [tese]. Belo Horizonte (MG): Universidade Federal de Minas Gerais, 2011.

27. Foglia Manzillo V, Oliva G, Pagano A, Manna L, Maroli M, Gradoni L. Deltamethrin-impregnated collars for the control of canine leishmaniasis: evaluation of the protective effect and influence on the clinical outcome of Leishmania infection in kennelled stray dogs. Vet Parasitol. 2006 Nov;142(1-2):142-5.

28. Coura-Vital W, Reis AB, Fausto MA, Leal GG, Marques MJ, Veloso VM, et al. Risk factors for seroconversion by Leishmania infantum in a cohort of dogs from an endemic area of Brazil. PLoS One. 2013 Aug;8(8):e71833.

29. Barata RA, Peixoto JC, Tanure A, Gomes ME, Apolinário EC, Bodevan EC, et al. Epidemiology of visceral leishmaniasis in a reemerging focus of intense transmission in Minas Gerais State, Brazil. Biomed Res Int. 2013 Aug;2013:405083.

30. Killick-Kendrick R, Killick-Kendrick M, Focheux C, Dereure J, Puech MP, Cadiergues MC. Protection of dogs from bites of phlebotomine sandflies by deltamethrin collars for control of canine leishmaniasis. Med Vet Entomol. 1997 Apr;11(2):105-11.

\section{Resumen}

objetivo: describir las dificultades operativas en la implementación de los collares impregnados con deltametrina para el control de la leishmaniasis visceral. Métodos: ensayo comunitario de intervención en la municipalidad de Montes Claros, MG, Brasil, comparando (i) área control - perros sin collares - y (ii) área de intervención - uso de collares impregnados con deltametrina $4 \%$-; se realizó una encuesta serológica inicial, seguida de otros tres ciclos (a los 12, 18 y 24 meses). Resultados: de 4.388perros inicialmente seronegativosy con los collares, 36,9\% no participaron del segundo ciclo, $27,0 \%$ se perdieron por desaparición/donación/venta y 22,6\% porque el domicilio estaba cerrado; la pérdida de los collares fue del 56,1\% en un año; entre los perros que permanecieron más tiempo en estudio, la pérdida fue menor. Conclusión: alta frecuencia de pérdida de los collares y de domicilios cerrados son dificultades operativas para la implementación de un programa nacional de control basado en la estrategia evaluada.

Palabras clave: Leishmaniasis Visceral; Leishmania infantum; Perros; Prevención de Enfermedades; Estudios de Seguimiento.

Recebido em 02/01/2017

Aprovado em 28/05/2018 